$\xi=-$ 国

\title{
Application of the Discrete Laguerre Wavelet Transform
}

\author{
Bavanari Satyanarayana*, Aama Abdulelah \\ Department of Mathematics, Acharya Nagarjuna University, Andhra Pradesh, India-522510 \\ *Corresponding author E-mail: drbsn63@yahoo.co.in ; Tel+91-9182755524
}

\begin{abstract}
In this work, Discrete Laguerre Wavelet Transform (DLWT) was used in the processing of images where they were divided into blocks and each block dimension is equal to matrix dimension obtained from DLWT. The concepts Peak Signal to Noise Ratio and Mean Squared Error were used. The examples used to prove the efficiency of the proposed method where good and convincing accounts were obtained.
\end{abstract}

Keywords: Discrete Laguerre Wavelet Transform (DLWT), Peak Signal to Noise Ratio (PSNR), Mean Squared Error (MSE), Image processing, .

\section{Introduction}

A digital image is a rectangular array of dots or picture elements, arranged in $m$ rows and $n$ columns. The expression $m \times n$ is called the resolution of the image and the dots are called pixels [1], [2]. The importance of images is shown to be an important source of information through image processing technology [8], [9]. Digital image noise removal involves visual systems, electronics technology [11], computer science and mathematical analysis. This science has a very complex edge, it can be used in many fields such as medicine and military systems [3], [4]. Most of the studies in the field of image treatment have been used many types of waveforms known as Haar or other, which has many qualities that qualify them to use in this field [10]. The current work is different from references [5], [6] and [7], where the matrices used in image processing, larger dimensions, Arithmetic Peak Signal to Noise Ratio (PSNR), Mean Squared Error (MSE), which all were not discussed in the a f o r e m e $n$ t i o n e d $r$ e f e $r$ e $n$ c e $s$.

\section{Applied Method}

Our proposed method is known from the previous work to use Laguerre wavelets in image processing using Matlab program which will help us to build new algorithms that show the stages in which the image will be processed and then return to the original image without loss, which we will touch through the results be proved in subsequent paragraphs.

\subsection{Laguerre Wavelets}

The following family function [5]

$$
\rho_{s, r}(t)=|s|^{\frac{-1}{2}} \rho\left(\frac{t-r}{s}\right) \quad s, r \in R, \quad s \neq 0
$$

where, $\rho(t)=\left[\rho_{\mathrm{o}}(t), \rho_{1}(t), \ldots ., \rho_{\mathrm{M}-1}(t)\right]^{\mathrm{T}}$. The elements on $[0,1]$. Laguerre wavelet has four arguments $\mathrm{k}=1,2, \ldots, \mathrm{n}=1$, $2, \ldots, 2^{k-1}, m$ is order for Laguerre polynomials and $t$ is normalized time. If we dilation by parameter $s=2^{-(k+1)}$ and translation by parameter $r=2^{-(k+1)}(2 n-1)$ and use transform

$x=2^{-(k+1)}\left(2^{k} t\right)$ in (1). Then we obtain the following equation

$\rho_{n, m}(t)=\left\{\begin{array}{cc}2^{k+1 / 2} \tilde{L}_{m}\left(2^{k} t-2 n+1\right) & \frac{n-1}{2^{k-1}} \leq t \leq \frac{n}{2^{k-1}} \\ 0 & \text { otherwise }\end{array}\right.$

where $\quad \tilde{L}_{m}=\frac{1}{m !} L_{m} \quad$ for $\mathrm{k}=2$.

In equation (2), for $M=3$ the six basis functions are given by

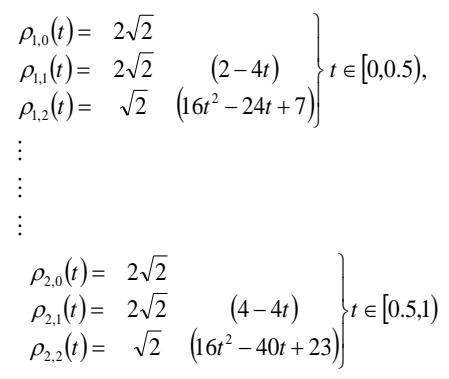

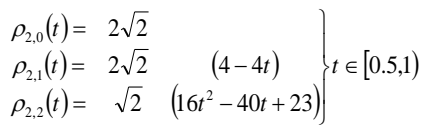

An approximation function $f(t) \in L^{2}[0,1]$ may be expanded as,

$f(t)=\sum_{n=1}^{\infty} \sum_{m=0}^{\infty} A_{n, m} \rho_{n, m}(t)$

where

$A_{n, m}=\left\langle f(t), \rho_{n, m}(t)\right\rangle$

$\rho_{o}(t), \rho_{1}(t), \ldots, \rho_{M-1}(t)$, are the basis functions, orthogonal 
In equation (4), $\langle. .$,$\rangle denotes the inner product with weight func-$ tion $w_{n}(t)$ on the Hilbert Space $[1,0)$. If the infinite series in the above equation is truncated then equation (3) can be written as

$$
f(t)=\sum_{n=1}^{k^{k-1}} \sum_{m=0}^{M-1} A_{n, m} \rho_{n, m}(t)=A^{T} \rho_{n, m}(t)
$$

where $A$ and $\rho(t)$ are $2^{k-1} M \times 1$ matrices given by:

$$
\begin{aligned}
A & =\left[A_{1,0}, A_{1,1}, \ldots, A_{2,0}, \ldots, A_{2,(M-1)}, \ldots, A_{2^{k-1}, 0}, \ldots, A_{2^{k-1}, M-1}\right]^{T} \\
\rho(t) & =\left[\begin{array}{l}
\rho_{1,0}, \rho_{1,1}(t), \ldots, \rho_{1, M-1}(t), \rho_{2,0}(t), \ldots, \\
\rho_{2^{k-1}, M-1}(t), \ldots, \rho_{2^{k-1}, 0}(t), \ldots, \rho_{2^{k-1}, M-1}
\end{array}\right]^{T}
\end{aligned}
$$

\subsection{Laguerre Wavelets with its Coefficients}

In this section, the coefficients of Discrete Laguerre wavelets transformations $(\mathrm{DLWT})_{\mathrm{C}}$ will be employed and displayed as matrix and derived from [5], [6] and [7]. Initially we use two dimensional matrix $(8 \times 8)$. The following matrix is the coefficients of DLWT are obtained from convolution theorem:

$\lg w=\left[\begin{array}{cccccccc}2 & 0 & 0 & 0 & 0 & 0 & 0 & 0 \\ 2 & -2 & 0 & 0 & 0 & 0 & 0 & 0 \\ 2 & -4 & 1 & 0 & 0 & 0 & 0 & 0 \\ 2 & -6 & 3 & \frac{-1}{3} & 0 & 0 & 0 & 0 \\ 2 & -8 & 6 & \frac{-4}{3} & \frac{1}{12} & 0 & 0 & 0 \\ 2 & -10 & 10 & \frac{-10}{5} & \frac{5}{12} & \frac{-1}{60} & 0 & 0 \\ 2 & -12 & 15 & \frac{-20}{3} & \frac{15}{12} & \frac{-6}{60} & \frac{1}{360} & 0 \\ 2 & -14 & 21 & \frac{-35}{5} & \frac{5}{12} & \frac{-21}{60} & \frac{7}{365} & \frac{-1}{2520}\end{array}\right]$

$\operatorname{lgw} 1$ is the matrix $\operatorname{lgw}$ with norm $\frac{1}{\sqrt{2}}$ (see [6])

$\lg w 1=\left[\begin{array}{cccccccc}1.4 & 0 & 0 & 0 & 0 & 0 & 0 & 0 \\ 1.4 & -1.4 & 0 & 0 & 0 & 0 & 0 & 0 \\ 1.4 & -2.8 & 0.71 & 0 & 0 & 0 & 0 & 0 \\ 1.4 & -4.2 & 2.12 & -0.2 & 0 & 0 & 0 & 0 \\ 1.4 & -5.7 & 4.24 & -0.9 & 0.1 & 0 & 0 & 0 \\ 1.4 & -7.1 & 7.07 & -2.4 & 0.3 & -0.012 & 0 & 0 \\ 1.4 & -8.5 & 10.6 & -4.7 & 0.9 & -0.071 & 0.002 & 0 \\ 1.4 & -9.9 & 14.8 & -8.2 & 0.3 & -0.247 & 0.014 & -0.000281\end{array}\right]$

The above matrix will be used to process the reference. The following example shows this conversion.

\section{Example 2.2.1}

The following image data illustrates above correlated pixels. The following sequence of values gives the intensity of 64 adjacent pixels in a row of a continuous-tone image original signal (8x8 matrix)

$\mathrm{AR}=\operatorname{randi}(100,[8$ 8 $])$

$$
A R=\left[\begin{array}{cccccccc}
63 & 70 & 74 & 60 & 67 & 6 & 10 & 85 \\
70 & 43 & 44 & 46 & 51 & 16 & 91 & 92 \\
73 & 84 & 70 & 6 & 22 & 2 & 11 & 99 \\
35 & 74 & 95 & 23 & 58 & 44 & 52 & 51 \\
52 & 37 & 79 & 84 & 13 & 84 & 15 & 28 \\
56 & 46 & 71 & 2 & 68 & 62 & 56 & 11 \\
16 & 39 & 11 & 87 & 60 & 53 & 1 & 51 \\
57 & 78 & 39 & 8 & 6 & 87 & 77 & 59
\end{array}\right]
$$

The matrix of transform image

$\mathrm{RR}=\mathrm{AR} * \lg \mathrm{w}$;

$$
R R=\left[\begin{array}{cccccccc}
615.183 & -1910.6 & 1874.5 & -839.8 & 39.5 & -21.8 & 1.1 & 0.0 \\
640.639 & -2464.9 & 2789.5 & -1284.5 & 115.2 & -29.3 & 1.4 & 0.0 \\
519.016 & -1554.2 & 1756.4 & -895.4 & 40.7 & -25.3 & 1.3 & 0.0 \\
610.94 & -2056.2 & 1982.0 & -829.6 & 77.3 & -16.8 & 0.8 & 0.0 \\
554.372 & -1704.1 & 1458.0 & -531.7 & 47.0 & -8.9 & 0.4 & 0.0 \\
526.087 & -1681.4 & 1538.6 & -565.4 & 75.0 & -7.4 & 0.2 & 0.0 \\
449.72 & -1682.9 & 1589.5 & -627.4 & 35.0 & -13.3 & 0.7 & 0.0 \\
581.242 & -2141.1 & 2378.0 & -1062.3 & 111.4 & -21.0 & 0.9 & 0.0
\end{array}\right]
$$

The matrix of inverse $\left(\mathrm{C}_{\mathrm{s}} \mathrm{DLWT}\right)$

$\operatorname{Lgv}=\operatorname{inv}(\lg w 1)$

$\lg v=\left[\begin{array}{cccccccc}0.70711 & 0 & 0 & 0 & 0 & 0 & 0 & 0 \\ 0.70711 & -0.71 & -8 \times 10^{-13} & 3 \times 10^{-15} & -3 \times 10^{-15} & 1 \times 10^{-15} & -1 \times 10^{-16} & -1 \times 10^{-17} \\ 1.41421 & -2.83 & 1.41421 & 5 \times 10^{-15} & -6 \times 10^{-15} & 3 \times 10^{-15} & -6 \times 10^{-16} & 1 \times 10^{-16} \\ 4.24264 & -12.7 & 12.727922 & -4.24264 & -5 \times 10^{-14} & 1 \times 10^{-14} & -3 \times 10^{-15} & 0 \\ 16.9706 & -67.9 & 101.82338 & -67.8823 & 16.9705627 & 1 \times 10^{-13} & -2 \times 10^{-14} & -8 \times 10^{-15} \\ 84.8528 & -424 & 848.52814 & -848.528 & 424.264069 & -84.852814 & -3 \times 10^{-13} & -7 \times 10^{-15} \\ 509.117 & -3055 & 7636.7532 & -10182.3 & 7636.75324 & -3054.7013 & 509.116882 & -2 \times 10^{-13} \\ -103351 & 4 e^{+05} & -566647.09 & 302924.5 & 17819 . .0909 & -74840.182 & 2494.7272 & -3563.81818\end{array}\right]$

The following matrix shows the results of the example 2.2.1 Inverse Matrix of AR2 = round $\left(R^{*}\right.$ $\left(\mathrm{IC}_{\mathrm{s}} \mathrm{DLW}\right)=\mathrm{AR}$

$A R 2=\left[\begin{array}{cccccccc}63 & 70 & 74 & 60 & 67 & 6 & 10 & 85 \\ 70 & 43 & 44 & 46 & 51 & 16 & 91 & 92 \\ 73 & 84 & 70 & 6 & 22 & 2 & 11 & 99 \\ 35 & 74 & 95 & 23 & 58 & 44 & 52 & 51 \\ 52 & 37 & 79 & 84 & 13 & 84 & 15 & 28 \\ 56 & 46 & 71 & 2 & 68 & 62 & 56 & 11 \\ 16 & 39 & 11 & 87 & 60 & 53 & 1 & 51 \\ 57 & 78 & 39 & 8 & 6 & 87 & 77 & 59\end{array}\right]$

$\mathrm{ERROR}=(\mathrm{AR}-\mathrm{AR} 2)=0$

The following program shows above matrices

$$
\begin{aligned}
& \operatorname{Lgw} 1=(1 / \mathrm{sqrt}(2)) * \operatorname{lgw} ; \\
& \text { ar=randi(100,[8 8]); } \\
& \text { rr=ar*lgw1; } \\
& \operatorname{lgv}=\operatorname{inv}(\operatorname{lgw} 1) ; \\
& \text { rr2=round(rr*lgv); } \\
& \text { d=ar-rr2 }
\end{aligned}
$$

$\mathrm{ERROR}=(\mathrm{AR}-\mathrm{AR} 2)=0$ 
Through the above example, we can formulate the following algorithm and Figure 1 shows the processing of algorithm 2.2.2.

\section{Algorithm 2.2.2}

Theoretical efficiency by using MATLAB

In put :- Matrix of $(8 \times 8)$.

Out Put :- processing of matrix by using ( $8 \times 8$ ) DLWT

Step 1- Matrix of Coefficients of DLWT

Step 2- Matrix of $\mathbf{8} \times \mathbf{8}=\mathrm{AR}$

$\mathrm{AR}=\operatorname{randi}(100,[8 \mathrm{8}])$;

Step 3- Transformation of the AR=RR.

$\mathrm{RR}=\mathrm{AR} * \mathrm{C}_{\mathrm{s}} \mathrm{DLWT}$

Step 4- Inverse $\left(\mathrm{C}_{\mathrm{S}} \mathrm{DLWT}\right)$.

$\mathrm{IC}_{\mathrm{s}} \mathrm{DLWT}=\operatorname{inv}\left(\mathrm{C}_{\mathrm{s}} \mathrm{DLWT}\right)$;

Step 5- Inverse Transformation of the RR2=AR.

Step 6- Error of this process equal zero.

End

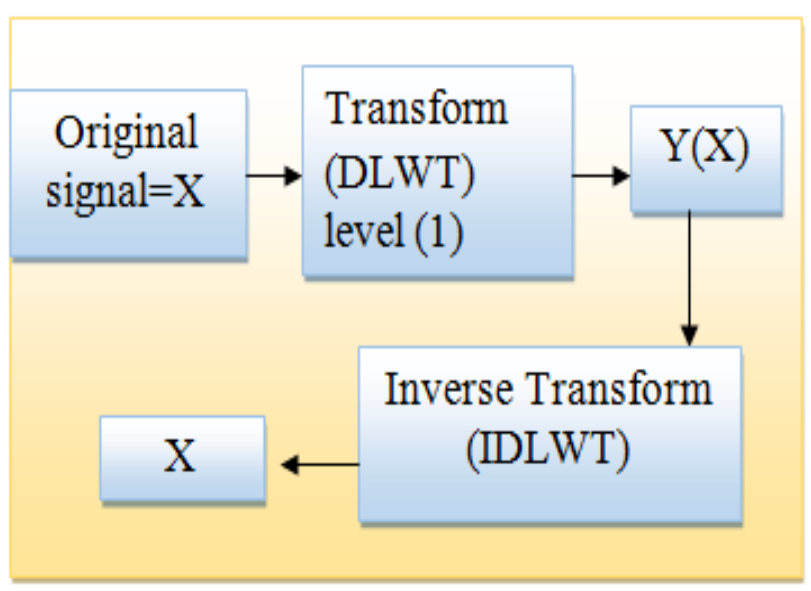

Fig.1: Transformation of DLWT level 1

The similar process that was done in example 2.2.1 will apply it to a wider glossy and we get a good result which makes us consider our theory fast and suitable for the treatment of the image.

\section{Example 2.2.3}

The following image data illustrates what can be done with correlated pixels. The following sequence of values gives the intensities of 256 adjacent pixels in a row of a continuous-tone image. Then we obtained

$$
\mathrm{ERROR}=(\mathrm{AR}-\mathrm{AR} 2)=0
$$

\section{Algorithm 2.2.4}

Theoretical efficiency by using MATLAB program. In put:- Matrix of $(16 \times 16)$.

Out put:- processing of matrix by using $(16 \times 16)$ (level (2) $\left(\mathrm{C}_{\mathrm{s}} \mathrm{DLWT}\right)$.

Steps:-

1. Matrix of Co-efficient of Laguerre wavelets Transform (16×16).

2. Matrix of $16 \times 16=\mathrm{AR}$

$\mathrm{AR}=\operatorname{randi}(100,[1616])$;

3. Transformation of the AR=RR.

$\mathrm{RR}=\mathrm{AR} * \mathrm{C}_{\mathrm{S}} \mathrm{DLWT}$

Inverse $\left(\mathrm{C}_{\mathrm{s}} \mathrm{DLWT}\right)$

$\mathrm{IC}_{\mathrm{s}} \mathrm{DLWT}=\operatorname{inv}\left(\mathrm{C}_{\mathrm{s}} \mathrm{DLWT}\right)$;

4. Inverse Transformation of the RR2=AR.

5. Errore of this process equal zero.

End
This program was represented in Figure 2.

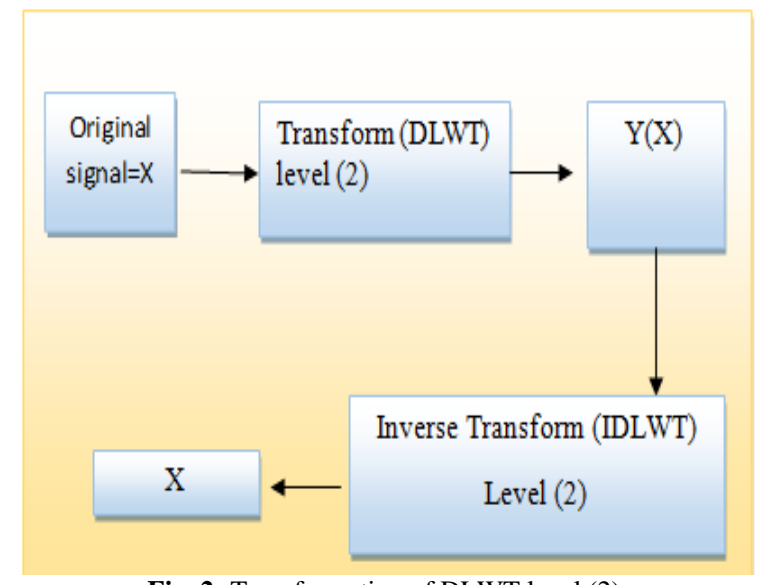

Fig. 2: Transformation of DLWT level (2)

\subsection{Image Processing By Using New Method}

From the previous example, we found that the processing process using the Discrete Laguerre Wavelet Transform (DLWT) where we obtained the amount of error is equal to zero, which shows that our proposed method, is suitable for the use of image processing and the development of programs for using Matlab, where it is installed and ready to use for many of the usual waveforms such as Haar Wavelet and others as seen in many.

The following Figure 3 shows that processing of the colored image converting to a gray image by using DLWT through MATLAB program.

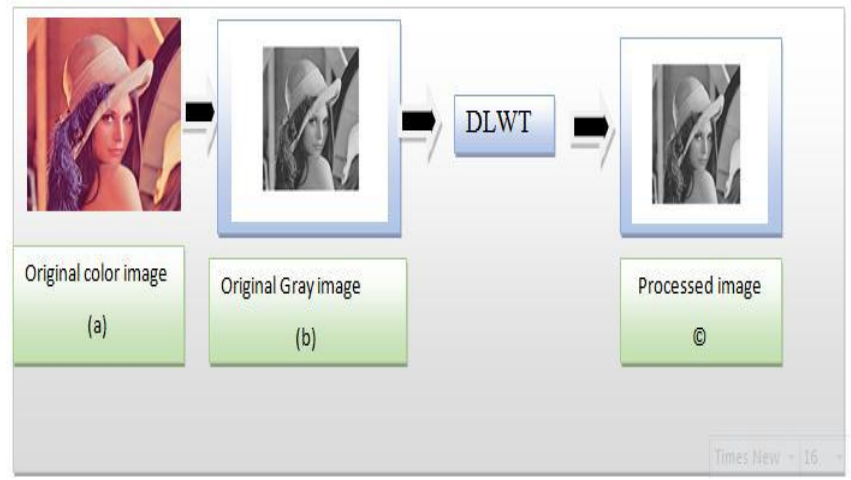

Fig. 3.: Processing of the colored image after converting it to a gray image using DLWT

\section{Example 2.3.1}

In this example, we process the image (jpg) by using DLWT and the matrix (lgw1) of coefficient DLWT level (1) and level (2) by Matlab program.

fn=uigetfile('*.jpg');

$\mathrm{im}=$ imread$(\mathrm{fn})$;

This proposed method shown in the Figure 4.

\author{
Algorithm 2.3.2. \\ Image processing by \\ Discrete Laguerre wavelet transform (DLWT) \\ level (1) \& level (2). \\ Input :- gray image \\ Output :- processed gray image
}




\section{Steps:-}

1. Load color image and converting it to a gray image

2. $\mathrm{fn}=$ uigetfile('*.jpg');

3. $\mathrm{im}=\mathrm{imread}(\mathrm{fn})$;

4. Converting it to a gray

5. Divided it to blocks every block has dimension (16 $\times 16)$.

6 . Then we begin to process block on its own by using

7. Discrete Laguerre wavelet transform (DLWT)

8. level (1) \& level (2).

9. Inverse Discrete Laguerre wavelet transform (IDLWT)

10. level (1) \& level (2).

11. End.

\section{Example 2.3.2}

In this example, we use without $\left(\frac{2}{\sqrt{2}}\right)$. The other part will be Normalization The following example will show us that situation and it is a case normalization
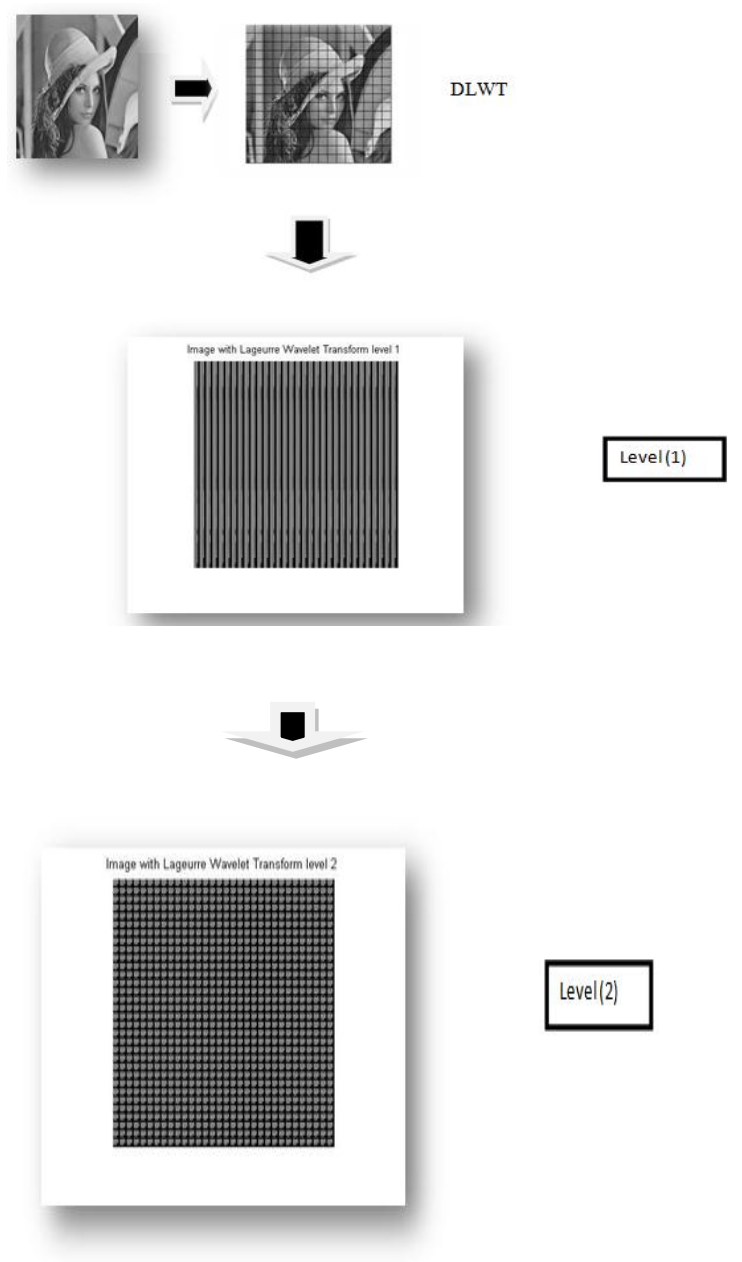

Fig. 4:. Processing of the gray image using DLWT

\section{Example 2.3.3}

Here, we show the normalization. Figure 5 shows that the process of the gray image using Matlab and DLWT to normalize level 1 and level 2.

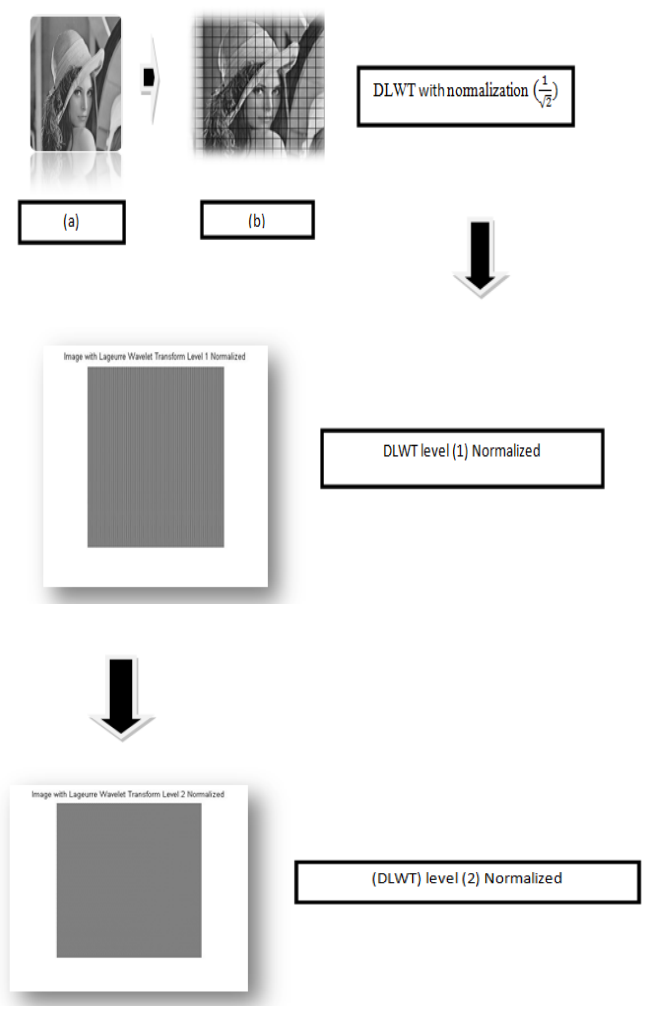

Fig. 5:. Processing of the gray image using DLWT normalized

\author{
Algorithm. 2.3.4 \\ Image processing by Normalized Discrete Laguerre Wavelet \\ Transform (DLWT) level (1) \& level (2). \\ Input :- gray image . \\ Output :- processed gray image \\ Steps:- \\ 1. Load color image and converting it to a gray image \\ fn=uigetfile('*.jpg'); \\ im=imread(fn); \\ 2. Converting it to a gray \\ 3. Divided it to blocks every block has dimension $(16 \times 16)$.
}

Then we begin to process block on its own by using Normalized Discrete Laguerre Wavelet Transform (DLWT) level (1) \& level (2).

4. Inverse Discrete Laguerre wavelet transform (IDLWT) level (1) \& level (2).

End

In the next section we will show the comparison between the original image and the processed image.

Peak Signal to Noise Ratio (PSNR)

PSNR $=10 * \log _{10}\left(\frac{255^{2}}{M S E}\right)$

Mean Squared Error (MSE)

MSE $=\frac{1}{M * N} \sum_{x=0}^{M-1} \sum_{y=0}^{N-1}\left(I_{x, y}-O_{x y}\right)^{2}$, where $I_{x, y}$ mis original signal and $O_{x, y}$ is after processing signal.

\section{Example 2.3.5}

In this example, we complete example 2.3.4 for calculate the value of Peak Signal to Noise Ratio.

The Figure 6 shows the algorithm 2.3.4 level 1 and Figure 7 shows the algorithm 2.3.4 for level 2 . 

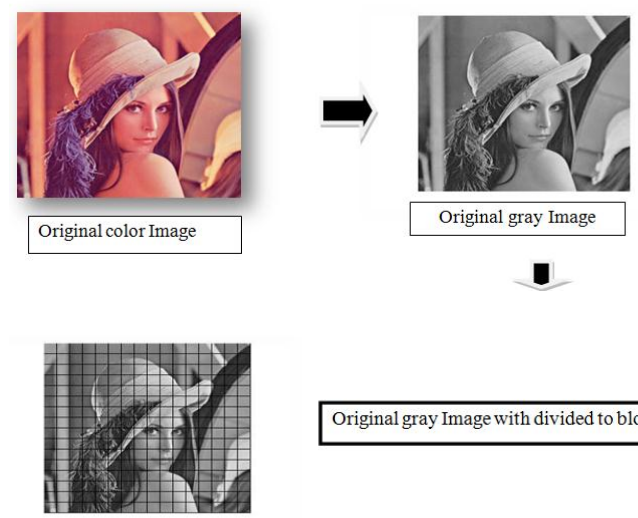

Original gray Image with divided to blocks

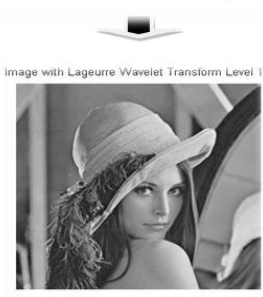

Image processing by DLWT

Fig .6: Discrete Laguerre Wavelet Transform (DLWT) Level (1)

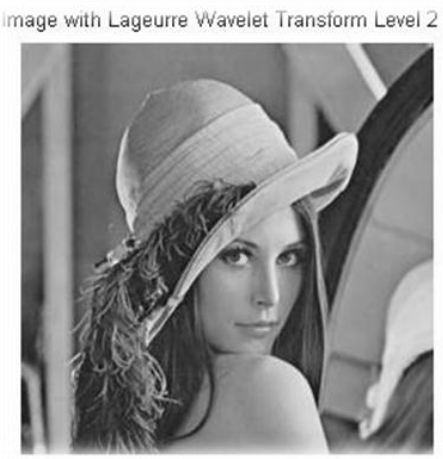

Fig. 7: Discrete Laguerre Wavelet Transform (DLWT) Level (2)

Information Retrieving

MSE $=4.3096 \mathrm{e}-23=4.3096 * 10^{-23}$

$\mathrm{PSNR}=271.7864$

\section{3 .Conclusion}

In this work, the use of Discrete Laguerre Wavelet Transform was used and the image was processed Convincing results were obtained. The results obtained a clearer picture. Moreover, the original picture did not lose its original characteristics. This was reached through Peak Signal to noise ratio and Mean Squared Error, where we concluded that the amount of loss is negligible.

\section{References}

[1] A. M. Raid, and W. M. Khedr, “ JPEG Image compression using discrete cosine transform- A survey", International Journal of Computer Sciences\& Engineering Survey (IJCSES) Vol 5, No 2, (2014), PP 39-47, arxiv.org/pdf/1405.6147.

[2] Arpita C. Raut, Dr. R. R Sedamkar, "Adaptive super- spatial prediction approach for lossless image compression' International Journal of Engineering research and Applications, Vol.4, (2014), pp.175-180, www.academia.edu/7676584/Adaptive_Super-Spatial.

[3] Abasi Julius, "Medical image compression by using discrete Haar Wavelet Transform", International Journal of Science and Research, Vol.6, No.14 (2013), pp:1196-1200.
[4] Ahmed Tariq Sadiq \& Abdulla Mohamed Awad, "Image compression method based on Wavelet and DCT transforms", Journal of Baghdad College of Economic Sciences, Vol.32, (2012), pp:11-21, www.iasj.net/iasj?func $=$ fulltext\&aId=72607

[5] B. Satyanarayana, Y. Pragathi Kumar, Asma Abdulelah, "Laguerre Wavelet and its Programming' , International Journal of Mathematics Trends and Technology, Vol.49, No.2, (2017), pp:129-137, www.ijmttjournal.org/archive/ijmtt-v49p516

[6] B. Satyanarayana, Y. Pragathi Kumar, Asma Abdulelah, "Image Processing by using discrete Laguerre Wavelets Transform (DLWT)", International Journal of Computer Applications, Vol.171, No. 7, (2017), pp:28-39.

[7] B. Satyanarayana, Asma Abdulelah, "Mathematical aspects of Laguerre Wavelets Transformation", Annals of Pure and Applied Mathematics, Vol.16, No.1, (2018), pp:53-61, www.researchgate.net/journal/2279-087X_Annals_of...

[8] Bhonde Nilesh, Shinde Sachin, "Image compression using discrete Wavelet Transform", International Journal of Computer Technology and Electronics Engineering, Vol.3, (2013), pp:85-89, www.aui.ma/sse-capstone-repository/pdf/spring2016/Image...

[9] Othman Khalifa, "Wavelet coding design for image data compression" International Arab Journal of Information Technology, Vol.2, N.2, (2005).pp:118-128, available online www.researchgate.net/publication/220413703...

[10] Sarvarinder Singh, Er. Sarabjil Singh, "Rio Based Medical Image Compression Using DCT and Haar Wavelet", International Journal of Advanced Research in Computer and Communication Engineering, Vol.4, (2015), pp:469-472.

[11] Sadiq Jassim Abou-Loukh, Jaleel Sadoon Jameel, "Compression of an ECG signal using mixed transforms", Journal of Engineering, Vol.20, No.6, (2014), pp:109-123, ww.iasj.net/iasj?func $=$ fulltext $\&$ aId $=89558$ 